\title{
沸腾与冷凝传热及强化
}

魏进家 ${ }^{1 *}$, 宇波 $2^{*}$

1. 西安交通大学化学工程与技术学院, 西安 710049;

2. 北京石油化工学院机械工程学院, 北京 102617

* 联系人, E-mail: jjwei@mail.xjtu.edu.cn; yubobox@vip.163.com

\section{Boiling and condensation heat transfer and enhancement}

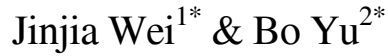 \\ ${ }^{1}$ School of Chemical Engineering and Technology, Xi'an Jiaotong University, Xi'an 710049, China; \\ ${ }^{2}$ School of Mechanical Engineering, Beijing Institute of Petrochemical Technology, Beijing 102617, China \\ *Corresponding authors, E-mail: jjwei@mail.xjtu.edu.cn; yubobox@vip.163.com \\ doi: 10.1360/TB-2020-0572
}

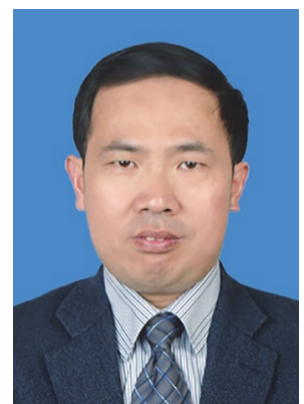

\section{魏进家}

西安交通大学化学工程与技术学院教授, 博士生导师. 1998 年毕业于西安交通大学 流体机械及工程专业, 获博士学位。从事 两相流与传热、太阳能光热与化学转化及 利用等研究. 2012 年获国家杰出青年科学 基金项目资助，2015 年入选教育部“长江 学者奖励计划”特聘教授.
沸腾与冷凝传热是伴随有巨大汽化潜热吸收和释放的相变对 流传热, 与无相变对流传热相比, 其换热系数具有数量级的差别, 是一种非常高效的传热方式. 沸腾包括池沸腾和流动沸腾, 而冷凝 包括膜状冷凝和珠状冷凝. 沸腾与冷凝传热在众多的工业过程中 均有广泛的应用, 如航空航天、电子工业、化工、机械制造、食品 加工、核能应用等领域。近年来，随着相关新技术的迅速发展，大 规模集成电路、微电子机械系统、大功率发光二极管以及核反应堆 等器件和设备面临着更高的散热要求, 由此导致的热失效问题严 重影响着器件和设备的工作性能和安全运行. 由于沸腾与冷凝强 化传热技术是解决该问题的潜在途径，其在国际上得到了越来越 多的关注和重视.

目前, 国内外的相关研究主要集中在两相传热机理和主-被动 沸腾与冷凝强化传热技术及其在工业中的应用等方面. 我国研究 者在沸腾与冷凝传热及强化研究中十分活跃, 成果丰硕. 为集中 展现我国研究者在该领域的研究成果, 促进同行间学术交流, 我 们组织了本期“沸腾与冷凝传热及强化”专辑, 内容涉及沸腾传热、 冷凝传热、两相传热数值计算及相变强化传热技术应用等, 主要集 中在从微观和介观层面揭示相变传热机理、采用表面微纳结构和 润湿性强化相变换热以及强化相变换热在平板热管中的应用三个 方面. 另外, 面向航天工程中变重力环境的相变换热应用, 在池沸 腾换热方面还涵盖了重力和变重力对沸腾换热性能影响及强化换 热内容. 专辑首先介绍了不同重力环境对沸腾换热性能影响的核 态池沸腾重力标度规律 ${ }^{[1]}$ 、梯度微纳结构多孔表面强化沸腾换热 ${ }^{[2]}$ 、 


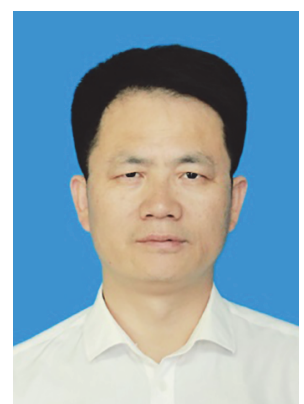

\section{宇波}

北京石油化工学院机械工程学院教授, 博 士生导师. 1999 年毕业于西安交通大学工 程热物理专业, 获博士学位. 从事计算流 体力学与传热学、油气长距离输送技术、 太阳能和地热能综合应用基础理论与技 术等研究. 2013 年获国家杰出青年科学基 金项目资助, 2016 年入选教育部“长江学 者奖励计划”特聘教授.
珠状冷凝机理模型及传热调控 ${ }^{[3]}$ 、介观相变传热数值模型及其在沸 腾和冷凝传热中的应用 ${ }^{[4]}$ 等方面的研究进展; 然后介绍了分子动 力学数值模拟在从微观上揭示表面润湿性对相变传热影响机理和 超临界流体类沸腾现象方面的研究成果 ${ }^{[5,6]}$; 最后呈现复合柱状微

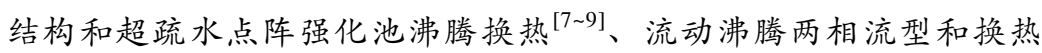
性能及歧管式微通道和多孔烧结表面强化流动沸腾换热 ${ }^{[10 ~ 12] ~}$ 以及 相变换热在矩形微通道型和丝网超薄型平板热管中的应用 ${ }^{[13,14]}$ 等 方面的最新研究成果.

本专辑的文章或是作者最新的研究进展, 或是多年来系统研 究的成果综述, 或是作者对该领域的理解与思考。文章从不同角度 和不同层面展示了作者对沸腾与冷凝传热及强化的思考与实践, 对本领域的研究热点与方向具有一定的代表性与指向性作用. 如 果本专辑的出版能够给读者以启发, 引发对沸腾与冷凝传热及强 化研究的兴趣, 为解决科研与应用中的相关问题提供一些启示, 那 将是本专辑作者和编者莫大的荣幸.

\section{参考文献}

1 Du W F, Zhao J F. Gravity scaling law of heat transfer in nucleate pool boiling (in Chinese). Chin Sci Bull, 2020, 65: 1629-1637 [杜王芳, 赵建福. 核态池沸腾传热现象中的重力标度规律. 科学通报, 2020, 65: 1629-1637]

2 Mo D C, Luo J L, Wang Y Q, et al. Porous surfaces with structural gradient: Enhancing boiling heat transfer and its application in phase-change devices (in Chinese). Chin Sci Bull, 2020, 65: 1638-1652 [莫冬传, 罗佳利, 汪亚桥, 等. 梯度结构多孔表面强化沸腾及 其在相变器件中的应用. 科学通报, 2020, 65: 1638-1652]

3 Tang G H, Hu H W, Niu D, et al. Advances in vapor dropwise condensation heat transfer (in Chinese). Chin Sci Bull, 2020, 65: 1653-1676 [唐桂华, 胡浩威, 牛东, 等. 蒸汽珠状冷凝传热的研究进展. 科学通报, 2020, 65: 1653-1676]

4 Li Q, Yu Y, Tang S. Multiphase lattice Boltzmann method and its applications in phase-change heat transfer (in Chinese). Chin Sci Bull, 2020, 65: 1677-1693 [李庆, 余悦, 唐诗. 多相格子 Boltzmann 方法及其在相变传热中的应用. 科学通报, 2020, 65: 1677-1693]

5 Wang Y, Xu J L, Li W, et al. Molecular dynamics study of mechanism of density fluctuation in supercritical fluid (in Chinese). Chin Sci Bull, 2020, 65: 1694-1704 [王艳，徐进良，李文，等. 超临界流体密度波动机理的分子动力学模拟. 科学通报, 2020, 65: 1694-1704]

6 Wang S L, Shen Y, He X, et al. Suppression of Leidenfrost phenomenon of nanodroplets through an external electric field (in Chinese). Chin Sci Bull, 2020, 65: 1705-1714 [王硕林, 沈园, 何金金, 等. 电场抑制纳米液滴的 Leidenfrost 现象. 科学通报, 2020, 65: 1705-1714]

7 Liu B, Kong X, Wei J J, et al. Pool boiling heat transfer and its critical heat flux mechanism in short-term microgravity (in Chinese). Chin Sci Bull, 2020, 65: 1715-1722 [刘斌, 孔新, 魏进家, 等. 短时间微重力池沸腾换热及其临界热流密度机理. 科学通报, 2020, 65: 1715-1722]

8 Cao Z Z, Sun D L, Wei J J, et al. Boiling heat transfer by using the VOSET method based on unstructured grids (in Chinese). Chin Sci Bull, 2020, 65: 1723-1733 [曹志柱, 孙东亮, 魏进家, 等. 基于非结构化 VOSET 方法的沸腾传热. 科学通报, 2020, 65: 1723-1733]

9 Wang X W, Wang H. Nucleate boiling on thin copper wires with micropatterned superhydrophobic and hydrophilic areas (in Chinese). Chin Sci Bull, 2020, 65: 1734-1740 [王新巍, 王吴. 带有超疏水点阵的微细铜丝上的核态沸腾. 科学通报, 2020, 65: 1734-1740]

10 Lu Y N, Wang D H, Zhao L, et al. Two-phase flow pattern and heat transfer coefficients of R245fa/R134a under non-uniform heat flux (in Chinese). Chin Sci Bull, 2020, 65: 1741-1751 [卢雅妮, 汪大海, 赵力, 等. 非均匀热流下 R245fa/R134a 两相流型及换热特性. 科学 通报, 2020, 65: 1741-1751]

11 Li W, Luo Y, Zhang J Z. Numerical simulation of subcooled flow boiling in a manifold microchannel heat sink (in Chinese). Chin Sci Bull, 2020, 65: 1752-1759 [李蔚, 骆洋, 张井志. 歧管式微通道热沉中过冷流动沸腾的数值模拟. 科学通报, 2020, 65: 1752-1759]

12 Cui F L, Hong F J, Lin T, et al. Distributed jet array impingement boiling on particle sintered porous surfaces (in Chinese). Chin Sci Bull, 2020, 65: 1760-1769 [崔付龙, 洪芳军, 林涛, 等. 烧结多孔表面分布式阵列射流沸腾. 科学通报, 2020, 65: 1760-1769]

13 Cui Z, Jia L, Huang D, et al. The visualized investigation on flow and heat transfer characteristics of flat-plate heat pipe with micro rectangular grooves (in Chinese). Chin Sci Bull, 2020, 65: 1770-1779 [崔卓, 贾力, 黄豆, 等. 矩形微槽道平板热管流动与传热特性可视 化. 科学通报, 2020, 65: 1770-1779]

14 Tang Y L, Dai X, Liu T Q, et al. Experimental research on structure parameters of the ultra-thin flattened heat pipe (in Chinese). Chin Sci Bull, 2020, 65: 1780-1790 [唐永乐, 代轩, 刘腾庆, 等. 丝网型超薄热管结构参数影响的实验探究. 科学通报, 2020, 65: 1780-1790] 